\title{
Treatment of sleep apnoea syndrome decreases cognitive decline in patients with Alzheimer's disease
}

\author{
Anne-Cécile Troussière, ${ }^{1,2,3}$ Christelle Monaca Charley, ${ }^{1,2,4}$ Julia Salleron, ${ }^{1,5}$ \\ Florence Richard, 1,5,6 Xavier Delbeuck, 1,2,3 Philippe Derambure, 1,2,4 \\ Florence Pasquier, ${ }^{1,2,3}$ Stéphanie Bombois ${ }^{1,2,3}$
}

\begin{abstract}
- Additional material is published online only. To view please visit the journal online (http://dx.doi.org/10.1136/ jnnp-2013-307544).

For numbered affiliations see end of article.
\end{abstract}

\section{Correspondence to} Dr Stéphanie Bombois, Memory Clinic, Roger Salengro Hospital, Rue Emile Laine, Lille F-59037, France; stephanie.bombois@chru-lille.fr

A-CT and CMC contributed equally to this study.

Received 3 January 2014 Revised 20 March 2014 Accepted 22 April 2014 Published Online First 14 May 2014
CrossMark

\begin{tabular}{|l|}
\hline To cite: Troussière A-C, \\
Monaca Charley C, \\
Salleron J, et al. J Neurol \\
Neurosurg Psychiatry \\
2014;85:1405-1408. \\
\hline
\end{tabular}

\section{ABSTRACT}

Background It is essential to detect and then treat factors that aggravate Alzheimer's disease (AD). Here, we sought to determine whether or not continuous positive airway pressure (CPAP) therapy for sleep apnoea syndrome (SAS) slows the rate of cognitive decline in mild-to-moderate $A D$ patients.

Methods Between January 2003 and June 2011, we included consecutive, mild-to-moderate $A D$ patients (a Mini Mental State Examination (MMSE) score at inclusion $\geq 15$ ) with severe SAS as determined by videopolysomnography (an apnoea-hypopnoea index $\geq 30$ ). In this single-blind, proof-of-concept trial, we analysed the mean decline in the annual MMSE score (the main outcome measure) according to whether or not the patients had received CPAP therapy. The decline was computed for each patient and for the first 3 years of follow-up.

Results of the 23 included patients, 14 underwent CPAP treatment. The CPAP and non-CPAP groups did not differ significantly in terms of their demographic characteristics or MMSE score at baseline. The median annual MMSE decline was significantly slower in the CPAP group $(-0.7(-1.7 ;+0.8))$ than in the non-CPAP group $(-2.2(-3.3 ;-1.9) ; p=0.013)$.

Conclusions In this pilot study, CPAP treatment of severe SAS in mild-to-moderate AD patients was associated with significantly slower cognitive decline over a three-year follow-up period. Our results emphasise the importance of detecting and treating SAS in this population.

\section{INTRODUCTION}

In the absence of an effective treatment for Alzheimer's disease (AD), it is essential to detect and treat factors that aggravate this condition (eg, vascular risk factors). ${ }^{1}$ Sleep apnoea syndrome (SAS) is associated with chronic, intermittent hypoxia and, in turn, an exacerbation of vascular risk factors. Recent data show that hypoxia interacts with the amyloidogenic process in $\mathrm{AD}$ in vitro, ${ }^{2}$ and in vivo in transgenic mice. ${ }^{3}$ However, to the best of our knowledge, the potentially harmful effect over time of SAS and chronic intermittent hypoxia on cognitive function in $\mathrm{AD}$ has not previously been studied. In a prospective, nonrandomised, single-blind, proof-of-concept study, we sought to determine whether continuous positive airway pressure (CPAP) treatment of severe SAS could slow the rate of cognitive decline in patients with mild-to-moderate AD. We considered that it would be ethically contentious to perform a randomised, double-blind, placebo-controlled trial over the period of time required to determine an effect on cognitive decline due to $\mathrm{AD}$, since CPAP treatment for severe SAS has a positive effect on excessive daytime sleepiness, quality of life and blood pressure. $^{4}$

\section{METHODS}

Patients

Consecutive patients monitored at the Lille Memory Clinic between January 2003 and June 2011 were included in the study if they met the following criteria: (1) probable mild-to-moderate $\mathrm{AD}$ dementia diagnosed by a multidisciplinary board according to the National Institute on Aging and the Alzheimer's Association guidelines, ${ }^{5}$ with a Mini Mental State Examination (MMSE) ${ }^{6}$ score $\geq 15$ at the time of the video-polysomnography (PSG) assessment and (2) severe SAS diagnosed by $\mathrm{PSG}^{7}$ on the basis of an apnoea-hypopnoea index (AHI) $\geq 30$ and suggestive clinical symptoms. ${ }^{8}$ We used the Epworth Sleepiness Scale (ESS) to measure daytime sleepiness. ${ }^{9}$

We excluded patients with neurological diseases other than $\mathrm{AD}$ and those with significant behavioural disorders.

\section{AD biomarkers}

In accordance with the French national guidelines on the diagnosis of dementia, routine MRI scans (1.5 Tesla) (or CT scans, if MRI was contraindicated) were systematically performed. Sandwich ELISAs (Innogenetics, Belgium) for cerebrospinal fluid (CSF) levels of T-tau, P-tau, and A $\beta-42$ proteins, and functional imaging (HMPAO $\mathrm{Tc}^{99 \mathrm{~m}}$ SPECT and/or fluorodeoxyglucose $\left.\left({ }^{18} \mathrm{FDG}\right) \mathrm{PET}\right)$ were performed for some patients. The biomarkers were considered as positive for an $\mathrm{AD}$ process when one or more following abnormalities were observed: medial temporal lobe atrophy on MRI, cortical temporoparietal hypometabolism on ${ }^{18}$ FDG-PET or hypoperfusion on HMPAO $\mathrm{Tc}^{99 \mathrm{~m}}$ SPECT, and decreased CSF levels of A $\beta-42$ and increased CSF levels of tau or P-tau. ${ }^{5}$ In patients who meet the core clinical criteria for probable 
$\mathrm{AD}$, biomarker evidence increases the certainty that the clinical dementia syndrome is indeed due to the AD process. ${ }^{5}$

\section{CPAP treatment}

Our patients were treated with self-titrating CPAP therapy. Two groups of $\mathrm{AD}$ patients with severe SAS were analysed: those treated with CPAP with good compliance for at least 3 months (the CPAP group) and those who had refused CPAP treatment or with a poor compliance (the non-CPAP group). Good compliance was defined as use of the CPAP device for more than $4 \mathrm{~h}$ a night and for more than five nights per week, on average. ${ }^{10}$ The duration of CPAP machine use was recorded at each visit to the Sleep Unit.

\section{Data analysis}

This was a single-blind, observational, proof-of-concept study. Cognitive and sleep data were collected prospectively. The primary outcome was the mean annual decline in the MMSE score. Monitoring with the MMSE started when AD and severe SAS were diagnosed at the baseline visit. The MMSE was subsequently administered at most every 6 months during routine follow-up visits. The duration of follow-up being heterogeneous, data were right-censured 4 years after the baseline visit.

\section{Statistical analysis}

Quantitative variables are quoted as the mean \pm SD or the median (range). Qualitative variables are quoted as number and percentage.

The CPAP and non-CPAP groups were compared using a $\chi^{2}$ test or Fisher's exact test (for qualitative variables) and a MannWhitney $U$ test (for quantitative variables), depending on the sample size and the normality of the data distribution.

The mean annual MMSE decline was computed for each patient. The respective outcomes in the two groups (CPAP vs non-CPAP) were compared in a Mann-Whitney $U$ test.

The threshold for statistical significance was set to $\mathrm{p}<0.05$. The statistical analysis was performed using SAS software (V.9.2, SAS Institute, Cary, North Carolina, USA).

\section{Ethics}

In view of the lack of randomisation, the independent ethics committee at Lille University Hospital considered that this prospective single-blind analysis was observational. The database was registered with the local data protection commission.

\section{RESULTS}

Between January 2003 and June 2011, 1144 patients with a MMSE score $\geq 15$ (mean MMSE at first visit: $22.5 \pm 4$; mean MMSE annual decline: $-2.1 \pm 4.8$; mean duration of follow-up: $4.1 \pm 3.1$ years) fulfilled the core clinical criteria for probable AD dementia. Sixty-six patients underwent PSG for suspected SAS on the basis of a sleep questionnaire administered by a neurologist at the Memory Clinic; 23 were diagnosed with severe SAS and were regularly monitored via the MMSE score (see figure 1). All these 23 patients had at least one positive biomarker for the $\mathrm{AD}$ pathological process (see the online supplementary table). Three patients displayed poor compliance with CPAP therapy and were included in the non-CPAP group. Hence, there were 14 $(60.9 \%)$ patients in the CPAP group and $9(39.1 \%)$ patients in the non-CPAP group. At baseline, there were no significant intergroup differences in terms of clinical data and MMSE score at baseline (see the table 1). In the CPAP group, 12 patients complete the ESS with a median score of $5(1 ; 16)$. Before CPAP therapy, two patients had a ESS score $>10$, without daytime

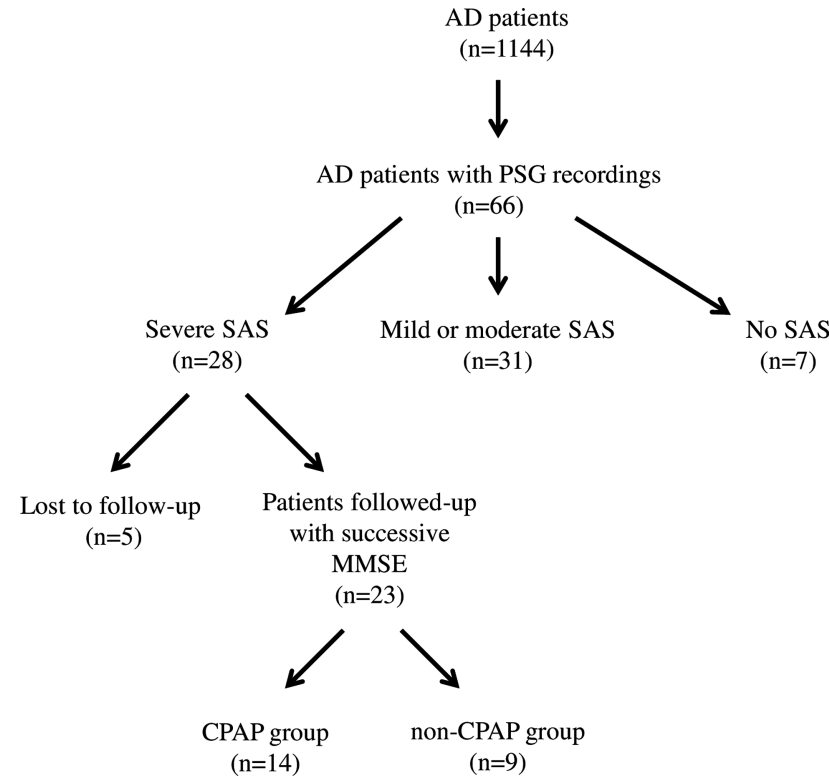

Figure 1 Flow chart (AD, Alzheimer's disease; CPAP, continuous positive airway pressure; PSG, polysomnography; SAS, sleep apnoea syndrome; CPAP group, AD patients with a severe SAS treated with CPAP; non-CPAP group, AD patients with a severe SAS without CPAP treatment).

sleepiness complaint after CPAP therapy. In the non-CPAP group, four patients completed the ESS with a median score of 6.5 (5; 10). All patients in the CPAP group had a good compliance over a median duration of use of $24.5(12 ; 46)$ months. The median remaining AHI in the CPAP group was $3(0.7 ; 11)$.

The median time interval between PSG and last MMSE was $3.3(1.8 ; 4.9)$ years. During this period, the two groups differed significantly in terms of the median annual cognitive decline $(-0.7(-1.7 ;+0.8)$ points per year in the CPAP group and -2.2 $(-3.3 ;-1.9)$ points per year in non-CPAP group $(p=0.013))$ (see figure 2). In the CPAP group, the median annual cognitive decline was not significantly different from $0(p=0.3757)$, and nine patients presented with an annual cognitive decline (from -0.3 to -9.8 points). In the non-CPAP group, the median annual cognitive decline was significantly different from 0 $(p=0.004)$, and all patients presented with an annual cognitive decline (from -1.4 to -7.7 points).

\section{DISCUSSION}

In this pilot study, probable $\mathrm{AD}$ patients treated with CPAP therapy for severe SAS displayed a significant slower annual MMSE decline than patients having refused CPAP therapy.

Given that (1) cognitive decline is not linear ${ }^{11}$ and (2) compliance with CPAP therapy changes as AD progresses, we chose to limit our analysis of the MMSE scores to the first 3 years of follow-up (ie, the median follow-up time).

In a recent randomised, double-blind, placebo-controlled trial, mild-to-moderate $\mathrm{AD}$ patients were randomised to either active CPAP therapy for 6 weeks or placebo CPAP therapy for 3 weeks and then active CPAP for 3 weeks. The study was not powered to detect interarm differences in cognitive changes but did show that 3 weeks of active CPAP therapy was associated with significant improvements in cognitive functioning. ${ }^{12}$ A subset of patients from this initial trial were followed-up (with five continuing CPAP therapy and five discontinuing CPAP therapy). The exploratory study found that the sustained use of 
Table 1 Baseline characteristics of the patients $(n=23)$

\begin{tabular}{|c|c|c|c|}
\hline & CPAP+ $(n=14)$ & CPAP- $(n=9)$ & $\mathrm{p}$ Value \\
\hline Age (years)* & $73.4(68 ; 79.6)$ & $77.6(74.8 ; 79.4)$ & 0.17 \\
\hline Gender ratio $\mathrm{H} / \mathrm{F}$ & $10 / 4$ & $4 / 5$ & 0.38 \\
\hline Educational level (0/1) & $6 / 8(42.9 \% / 57.1 \%)$ & $7 / 2(77.8 \% / 22.2 \%)$ & 0.19 \\
\hline MMSE at baseline* & $23.5(20 ; 27)$ & $20(16 ; 24)$ & 0.09 \\
\hline $\mathrm{AHI}^{*}$ & $47.7(36.7 ; 62.6)$ & $43.3(37.2 ; 51.2)$ & 0.52 \\
\hline BMI $\left(\mathrm{kg} / \mathrm{m}^{2}\right)^{*}$ & $26.7(24.7 ; 28.3)$ & $24.5(23.0 ; 33.1)$ & 0.81 \\
\hline $\begin{array}{l}\text { Vascular risk factor } \\
\text { (at least 1) }\end{array}$ & $10(71.4 \%)$ & 7 (77.8\%) & 1 \\
\hline Hypertension & $10(71.4 \%)$ & $7(77.7 \%)$ & 0.88 \\
\hline Diabetes & $2(14.3 \%)$ & $3(33.3 \%)$ & 0.57 \\
\hline Dyslipidaemia & $8(57.1 \%)$ & $6(66.6 \%)$ & 0.98 \\
\hline Smoking & $10(71.4 \%)$ & $3(33.3 \%)$ & 0.17 \\
\hline Heart disease & $9(20.5 \%)$ & $3(33.3 \%)$ & 0.3 \\
\hline Antihypertensive drugs & $8(57.1 \%)$ & $7(77.8 \%)$ & 0.39 \\
\hline Statin & $6(42.9 \%)$ & $5(55.6 \%)$ & 0.68 \\
\hline Antiplatelet agent & $8(57.1 \%)$ & $5(55.6 \%)$ & 1 \\
\hline Cholinesterase inhibitor & $13(92.8 \%)$ & $9(100 \%)$ & 0.81 \\
\hline Memantine & $9(64.2 \%)$ & $8(88 \%)$ & 0.40 \\
\hline $\begin{array}{l}\text { Duration of follow-up } \\
\text { (years) } \dagger\end{array}$ & $3.3(1.8 ; 3.6)$ & $3.0(2.3 ; 3.6)$ & 1 \\
\hline
\end{tabular}

${ }^{*}$ Median (range).

tMedian (IQR).

AD, Alzheimer's disease; AHI, apnoea hypopnoea index; BMI, Body Mass Index; educational level 0 , patients without any formal education or having completed only primary school education; educational level I, patients having completed middle school or high school education; CPAP-, patients who refused continuous positive airway pressure; CPAP+, patients who accepted continuous positive airway pressure; MMSE, Mini Mental State Examination; PSG, polysomnography; SAS, sleep apnoea syndrome.

CPAP therapy increased the likelihood of an improvement in executive functioning and psychomotor speed but was not associated with a significant effect on the rate of global cognitive decline (as evaluated by the MMSE). ${ }^{13}$ This conflict with our

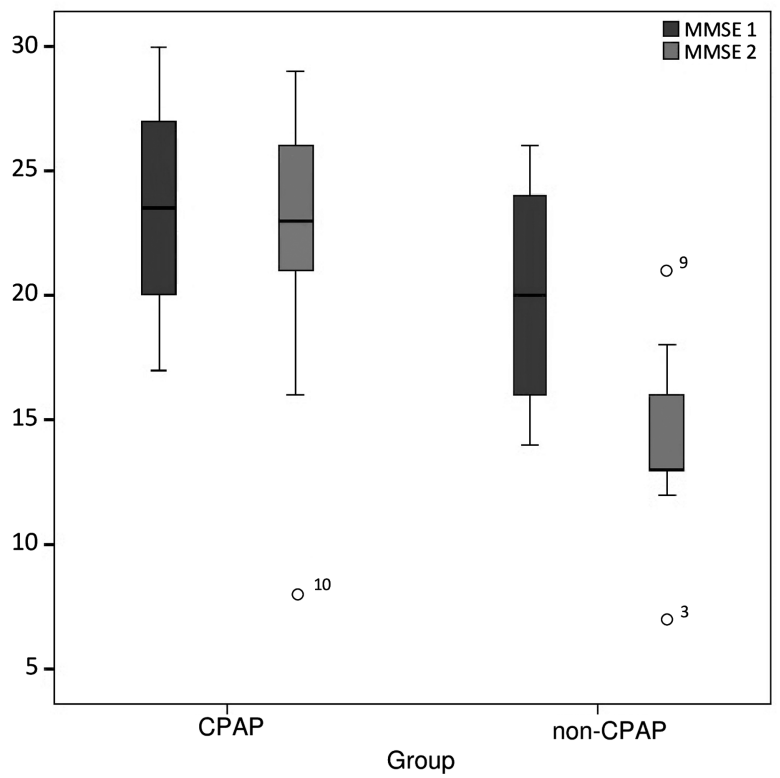

Figure 2 Mini Mental State Examination (MMSE) evolution in continuous positive airway pressure (CPAP) and non-CPAP groups during the follow-up (MMSE 1=MMSE at baseline, MMSE 2=last MMSE of the first 3 years of follow-up). findings may have been due to the smaller sample size, the shorter follow-up period $(13.3 \pm 5.2$ months $)$ and the less severe SAS syndrome $\left(\mathrm{AHI} \geq 10\right.$ ) in the study by Cooke et al, ${ }^{14}$ relative to the present study.

In non-demented patients, SAS is associated with impairments in attention-demanding cognitive functions attention, ${ }^{14}$ delayed long-term visual and verbal memory, visuospatial abilities and executive function, whereas language ability is unaffected..$^{15}$ CPAP treatment appears to improve executive dysfunction, memory, attention and global cognitive functioning. ${ }^{15}$ Hence, the less severe cognitive decline in our CPAP group may be due to a symptomatic effect of the correction of intermittent hypoxia.

The results of our observational study should be interpreted with caution, in view of the study design (lack of randomisation, double blinding or placebo control) and the non-adjustment of the statistical analyses with respect to the sample size. However, the intergroup difference in cognitive decline was robust. Our diagnoses of $\mathrm{AD}$ were well documented, with evidence of the $\mathrm{AD}$ process for most patients; this reinforces the value of our observation of global cognitive stability in the CPAP group. Moreover, the patients in the CPAP group did not differ significantly from those in the non-CPAP group in terms of demographic or clinical characteristics and the treatment of cardiovascular risk factors. The patients assessed with the ESS had no daytime sleepiness, suggesting no impact of daytime vigilance level on cognitive function. In the non-CPAP group, the mean annual decline in the MMSE was similar to that observed in our AD cohort $(n=1144)$ as a whole. The faster cognitive decline seen in the non-CPAP group might also be related to putative poor compliance with other treatments (medications for vascular risk factors, acetylcholinesterase inhibitors, etc.) and/or a lower MMSE score at baseline, relative to the CPAP group (even though the difference in the MMSE score at baseline was clearly not significant).

\section{CONCLUSION}

In view of the major public health issue raised by $A D$, our present work highlights the importance of SAS screening and CPAP treatment in mild-to-moderate $\mathrm{AD}$ patients, with a view to significantly slowing their cognitive decline. Our results must now be replicated in a larger, independent population of $\mathrm{AD}$ patients.

\section{Author affiliations}

${ }^{1}$ University Lille North of France, $\mathrm{CHU}$, Lille, France

${ }^{2}$ EA 1046, Lille, France

${ }^{3}$ Memory Clinic, Lille, France

${ }^{4}$ Department of Neurophysiology and Sleep Unit, Lille, France

${ }^{5}$ Department of Public Health and Biostatistic Unit, Lille, France

${ }^{6}$ INSERM UMR 744, Institut Pasteur, Lille, France

Acknowledgements The authors thank Nathalie Jourdan for help with database management.

Contributors A-CT: acquisition of data; analysis and interpretation; drafting the manuscript; critical revision of the manuscript for important intellectual content. CMC: study concept and design; acquisition of data; analysis and interpretation; drafting the manuscript; critical revision of the manuscript for important intellectual content. JS: statistical analysis; critical revision of the manuscript for important intellectual content. FR: study concept and design; Statistical analysis; critical revision of the manuscript for important intellectual content. XD: acquisition of data; critical revision of the manuscript for important intellectual content. PD: critical revision of the manuscript for important intellectual content. FP: analysis and interpretation; critical revision of the manuscript for important intellectual content. SB: study concept and design; analysis and interpretation; drafting the manuscript; critical revision of the manuscript for important intellectual content; study supervision. 
Funding This work has been developed and funded through the DISTALZ (Development of Innovative Strategies for a Transdisciplinary Approach to Alzheimer disease) laboratory of excellence, under the French governments 'Investments for the Future' programme.

Competing interests A-CT has (over the last 3 years) participated as a co-investigator in several therapeutic and diagnostic trials sponsored by pharmaceutical companies, including Roche and Pharnext. Honoraria were paid to the Association pour le Développement de la Recherche et de l'Innovation dans le Nord-Pas de Calais (Adrinord). CMC has received speaker's fees from Vitalaire and Resmed-Narval. XD participated in several therapeutic trials sponsored by pharmaceutical companies, including Bristol-Myers-Squibb, Noscira, Pfizer and Sanofi. FP has (over the last 3 years) participated in several therapeutic and diagnostic trials sponsored by pharmaceutical companies, including Bayer-Piramal, BMS, Eli-Lilly, Noscira, Pfizer and Sanofi. She has also served from time to time on scientific advisory boards for Janssen-Cilag, Eli-Lilly, Bayer, Novartis and Nutricia. Honoraria were paid to Adrinord. SB has (over the last 3 years) participated as co-investigator in several therapeutic and diagnostic trials sponsored by pharmaceutical companies, including Pfizer, Bristol-Myers-Squibb, Roche, Sanofi-Aventis, Noscira, GE-Healthcare, and Eli-Lilly. Honoraria were paid to Adrinord.

Ethics approval Internal Review Board of the Lille University Hospital.

Provenance and peer review Not commissioned; externally peer reviewed.

\section{REFERENCES}

1 Richard F, Pasquier F. Can the treatment of vascular risk factors slow cognitive decline in Alzheimer's disease patients? J Alzheimers Dis 2012;32:765-72.

2 Peers C, Dallas ML, Boycott HE, et al. Hypoxia and neurodegeneration. Ann N Y Acad Sci 2009;1177:169-77.

3 Zhang $X$, Zhou K, Wang R, et al. Hypoxia-inducible factor 1alpha (HIF-1alpha)mediated hypoxia increases BACE1 expression and beta-amyloid generation. J Biol Chem 2007;282:10873-80.
4 Brown DL, Anderson CS, Chervin RD, et al. Ethical issues in the conduct of clinical trials in obstructive sleep apnea. J Clin Sleep Med 2011;7:103-8.

5 McKhann GM, Knopman DS, Chertkow $\mathrm{H}$, et al. The diagnosis of dementia due to Alzheimer's disease: recommendations from the National Institute on Aging-Alzheimer's Association workgroups on diagnostic guidelines for Alzheimer's disease. Alzheimers Dement 2011;7:263-9.

6 Folstein MF, Folstein SE, McHugh PR. 'Mini-mental state'. A practical method for grading the cognitive state of patients for the clinician. J Psychiatr Res 1975;12:189-98.

7 Silber MH, Ancoli-Israel S, Bonnet MH. The visual scoring of sleep in adults. J Clin Sleep Med 2007;3:121-31.

8 Sleep-related breathing disorders in adults: recommendations for syndrome definition and measurement techniques in clinical research. The Report of an American Academy of Sleep Medicine Task Force. Sleep 1999:22:667-89.

9 Johns MW. A new method for measuring daytime sleepiness: the Epworth sleepiness scale. Sleep 1991;14:540-5.

10 Ghosh D, Allgar V, Elliott MW. Identifying poor compliance with CPAP in obstructive sleep apnoea: a simple prediction equation using data after a two week trial. Respir Med 2013;107:936-42.

11 Cortes F, Nourhashémi F, Guérin 0 , et al. Prognosis of Alzheimer's disease today: a two-year prospective study in 686 patients from the REAL-FR Study. Alzheimers Dement 2008;4:22-9.

12 Ancoli-Israel S, Palmer BW, Cooke JR, et al. Cognitive effects of treating obstructive sleep apnea in Alzheimer's disease: a randomized controlled study. J Am Geriatr Soc 2008;56:2076-81.

13 Cooke JR, Ayalon L, Palmer BW, et al. Sustained use of CPAP slows deterioration of cognition, sleep, and mood in patients with Alzheimer's disease and obstructive sleep apnea: a preliminary study. J Clin Sleep Med 2009;5:305-9.

14 Nemeth D, Csábi E, Janacsek K, et al. Intact implicit probabilistic sequence learning in obstructive sleep apnea. J Sleep Res 2012;21:396-401.

15 Bucks RS, Olaithe M, Eastwood P. Neurocognitive function in obstructive sleep apnoea: a meta-review. Respirology 2013;18:61-70. 